MATHEMATICS OF COMPUTATION

Volume 73, Number 246, Pages 853-860

S 0025-5718(03)01596-5

Article electronically published on July 29, 2003

\title{
A LOWER BOUND FOR RANK 2 LATTICE RULES
}

\author{
FRIEDRICH PILLICHSHAMMER
}

\begin{abstract}
We give a lower bound for a quality measure of rank 2 lattice rules
\end{abstract} which shows that an existence result of Niederreiter is essentially best possible.

\section{INTRODUCTION}

For the definition and the general theory of lattice rules for multivariate integration we refer to the monographs of Niederreiter [7] and of Sloan and Joe [9].

A rank 2 lattice rule is a quadrature rule for functions $f$ over the $s$-dimensional unit cube $[0,1]^{s}$ of the form

$$
Q(f)=\frac{1}{N} \sum_{k_{1}=1}^{n_{1}} \sum_{k_{2}=1}^{n_{2}} f\left(\left\{k_{1} \mathbf{z}_{1} / n_{1}+k_{2} \mathbf{z}_{2} / n_{2}\right\}\right),
$$

which cannot be re-expressed in an analogous form with a single sum. Here $n_{1}, n_{2}$ are positive integers such that $n_{2} \mid n_{1}, N=n_{1} n_{2}$ and $\mathbf{z}_{1}, \mathbf{z}_{2}$ are vectors in $\mathbb{Z}^{s}$. The integers $n_{1}, n_{2}$ are called the invariants of the lattice rule. (For a vector $\mathbf{x} \in \mathbb{R}^{s}$ the fractional part $\{\mathbf{x}\}$ is defined componentwise.)

For a given rank 2 lattice rule with invariants $n_{1}$ and $n_{2}, N=n_{1} n_{2}$ and with $\mathbf{z}_{1}=\left(z_{1}, \ldots, z_{s}\right)$ and $\mathbf{z}_{2}=\left(\zeta_{1}, \ldots, \zeta_{s}\right)$ for $z_{i}, \zeta_{i} \in \mathbb{Z}$, we define the quantity

$$
R_{N}\left(\mathbf{z}_{1}, \mathbf{z}_{2}\right):=\sum_{\substack{-N<h_{1}, \ldots, h_{s}<N \\ h_{1} z_{1}+\ldots+h_{s} z_{s} \equiv 0 \\ h_{1} \zeta_{1}+\ldots+h_{s} \zeta_{s} \equiv 0}}^{*\left(\bmod n_{1}\right)} \underset{1}{r\left(h_{1}\right) \ldots r\left(h_{s}\right)},
$$

where $\sum^{*}$ means summation over $\left(h_{1}, \ldots, h_{s}\right) \neq(0, \ldots, 0)$, and where $r(h)=$ $\max (1,|h|)$ for $h \in \mathbb{Z}$.

Let $f:[0,1]^{s} \longrightarrow \mathbb{R}$ be a real-valued periodic function with period 1 in each variable and with Fourier-coefficients $\hat{f}(\mathbf{h}), \mathbf{h}=\left(h_{1}, \ldots, h_{s}\right) \in \mathbb{Z}^{s}$, satisfying $|\hat{f}(\mathbf{h})|=$ $O\left(r(\mathbf{h})^{-\alpha}\right)$ for some $\alpha>1$ where $r(\mathbf{h})=\prod_{i=1}^{s} r\left(h_{i}\right)$. Then for the integration error of any rank 2 lattice rule (1) we have the relation

$$
\left|\int_{[0,1]^{s}} f(\mathbf{x}) d \mathbf{x}-Q(f)\right|=O\left(R_{N}\left(\mathbf{z}_{1}, \mathbf{z}_{2}\right)^{\alpha}\right) .
$$

For a proof of this result see [6] or [7].

Received by the editor August 5, 2002 and, in revised form, November 8, 2002

2000 Mathematics Subject Classification. Primary 11K06, 65D32, 41A55.

Key words and phrases. Rank 2 lattice rule, quadrature error bound.

Supported by the Austrian Research Foundation (FWF), project S 8305. 
Another reason for the importance of the quantity $R_{N}$ is its relation to the discrepancy $D_{N}$ of the finite $s$-dimensional point set

$$
\left\{\frac{k_{1}}{n_{1}} \mathbf{z}_{1}+\frac{k_{2}}{n_{2}} \mathbf{z}_{2}\right\}, \quad k_{i}=1, \ldots, n_{i}, \quad 1 \leq i \leq 2 .
$$

(For the definition of the discrepancy $D_{N}$ see, for example, 3] or 7].) In fact, it was shown by Niederreiter and Sloan [8] that the discrepancy of the point set (2) can be estimated by

$$
D_{N} \leq \frac{s}{N}+\frac{1}{2} R_{N}\left(\mathbf{z}_{1}, \mathbf{z}_{2}\right)
$$

(A proof of this estimate can also be found in [7].)

In [6] Niederreiter proved that for every dimension $s \geq 2$ and for any prescribed invariants $n_{1}$ and $n_{2}, N=n_{1} n_{2}$, there exist integer vectors of the form $\mathbf{z}_{1}=$ $\left(z_{1}, \ldots, z_{s}\right), \mathbf{z}_{2}=\left(0, \zeta_{2}, \ldots, \zeta_{s}\right)$ with $\operatorname{gcd}\left(z_{i}, n_{1}\right)=1,1 \leq i \leq s$, and $\operatorname{gcd}\left(\zeta_{i}, n_{2}\right)=1$, $2 \leq i \leq s$, such that

$$
R_{N}\left(\mathbf{z}_{1}, \mathbf{z}_{2}\right)<c_{s}^{\prime}\left(\frac{(\log N)^{s}}{N}+\frac{\log N}{n_{1}}\right),
$$

where $c_{s}^{\prime}>0$ is a constant only depending on $s$. Note that the lattice rule in Niederreiter's existence result is projection-regular. (See [7] for the definition of projection-regular lattice rules.)

In this paper we prove a lower bound for the quantity $R_{N}\left(\mathbf{z}_{1}, \mathbf{z}_{2}\right)$ which shows that Niederreiter's estimate is essentially best possible.

\section{Statement AND PROOF of the RESUlt}

We have

Theorem 2.1. For every dimension $s \geq 2$ there is a constant $c_{s}>0$, depending only on $s$, with the following property: for any prescribed invariants $n_{1}$ and $n_{2}$ with $n_{2} \mid n_{1}, N=n_{1} n_{2}$ and for any integer vectors $\mathbf{z}_{1}=\left(z_{1}, \ldots, z_{s}\right)$ and $\mathbf{z}_{2}=\left(\zeta_{1}, \ldots, \zeta_{s}\right)$ such that there is an index $1 \leq i_{0} \leq s$ with $\operatorname{gcd}\left(z_{i_{0}}, n_{1}\right)=1$, we have

$$
R_{N}\left(\mathbf{z}_{1}, \mathbf{z}_{2}\right)>c_{s} \frac{(\log N)^{s}}{N} \text {. }
$$

Remark 2.2. Note that by [7], Theorem 5.38] there is also a simple lower bound for $R_{N}\left(\mathbf{z}_{1}, \mathbf{z}_{2}\right)$ of the order $\left(\log n_{2}\right) / n_{1}$, which shows that the second term in Niederreiter's upper bound is essentially best possible.

Remark 2.3. In particular the lower bound for $R_{N}\left(\mathbf{z}_{1}, \mathbf{z}_{2}\right)$ from Theorem 2.1 is true for all projection-regular rank 2 lattice rules (see [7]), since by a result of Sloan and Lyness [10] a rank 2 lattice rule is projection-regular if and only if the vectors $\mathbf{z}_{1}, \mathbf{z}_{2} \in \mathbb{Z}^{s}$ can be chosen in such a way that $z_{1}=1, \zeta_{1}=0$ and $\zeta_{2}=1$. (Actually Sloan and Lyness give a characterization of projection-regular rank $r$ lattice rules.)

Remark 2.4. We note here that Larcher [4] proved the result stated in Theorem 2.1] for any rank 1 lattice rule, which shows that the existence theorems on good rank 1 lattice rules of Hlawka [1], Korobov [2] and Niederreiter [5] are best possible.

For the proof of Theorem[2.1] we need the following generalization of the Chinese remainder theorem: 
Lemma 2.5. Let $a_{1}, a_{2}, b_{1}, b_{2}, m_{1}, m_{2} \in \mathbb{Z}$ such that $\operatorname{gcd}\left(a_{i}, m_{i}\right) \mid b_{i}, 1 \leq i \leq 2$. Then the system of congruences

$$
a_{1} x \equiv b_{1} \quad\left(\bmod m_{1}\right), \quad a_{2} x \equiv b_{2} \quad\left(\bmod m_{2}\right)
$$

has a solution if and only if

$$
b_{1} a_{2}-b_{2} a_{1} \equiv 0 \quad(\bmod d),
$$

where $d:=\operatorname{gcd}\left(m_{1} m_{2}, m_{1} a_{2}, a_{1} m_{2}\right)$.

Proof. For $1 \leq i \leq 2$ let $d_{i}:=\operatorname{gcd}\left(a_{i}, m_{i}\right), a_{i}=\bar{a}_{i} d_{i}, b_{i}=\bar{b}_{i} d_{i}$ and $m_{i}=\bar{m}_{i} d_{i}$. Now since $b_{i} \equiv 0\left(\bmod d_{i}\right), 1 \leq i \leq 2$, we may divide the first congruence by $d_{1}$ and the second one by $d_{2}$ and our system of congruences becomes

$$
\bar{a}_{1} x \equiv \bar{b}_{1} \quad\left(\bmod \bar{m}_{1}\right), \quad \bar{a}_{2} x \equiv \bar{b}_{2} \quad\left(\bmod \bar{m}_{2}\right) .
$$

Since $\operatorname{gcd}\left(\bar{a}_{i}, \bar{m}_{i}\right)=1$, we can find $t_{i}$ such that $\bar{a}_{i} t_{i} \equiv 1\left(\bmod \bar{m}_{i}\right), 1 \leq i \leq 2$. Now we find that our system of congruences is equivalent to the system

$$
x \equiv \bar{b}_{1} t_{1} \quad\left(\bmod \bar{m}_{1}\right), \quad x \equiv \bar{b}_{2} t_{2} \quad\left(\bmod \bar{m}_{2}\right) .
$$

This system has a solution if and only if

$$
\bar{b}_{1} t_{1}-\bar{b}_{2} t_{2} \equiv 0 \quad\left(\bmod \operatorname{gcd}\left(\bar{m}_{1}, \bar{m}_{2}\right)\right) .
$$

From the definition of $t_{1}$ and $t_{2}$ we find that this congruence is equivalent to the congruence

$$
\bar{b}_{1} \bar{a}_{2}-\bar{b}_{2} \bar{a}_{1} \equiv 0 \quad\left(\bmod \operatorname{gcd}\left(\bar{m}_{1}, \bar{m}_{2}\right)\right) .
$$

Finally from the definition of $\bar{a}_{i}$ and $\bar{b}_{i}, 1 \leq i \leq 2$, this congruence is equivalent to

$$
b_{1} a_{2}-b_{2} a_{1} \equiv 0 \quad(\bmod d)
$$

with $d:=\operatorname{gcd}\left(m_{1}, a_{1}\right) \operatorname{gcd}\left(m_{2}, a_{2}\right) \operatorname{gcd}\left(\bar{m}_{1}, \bar{m}_{2}\right)$. Recalling the definition of $\bar{m}_{1}$ and $\bar{m}_{2}$, we have

$$
\begin{aligned}
d & =\operatorname{gcd}\left(\operatorname{gcd}\left(m_{2}, a_{2}\right) m_{1}, \operatorname{gcd}\left(m_{1}, a_{1}\right) m_{2}\right) \\
& =\operatorname{gcd}\left(m_{1} m_{2}, m_{1} a_{2}, a_{1} m_{2}\right)
\end{aligned}
$$

and we are done.

Proof of Theorem [2.1] W.l.o.g. we may assume that $z_{1}=1$. In the following let $\bar{n}_{1}:=n_{1} / n_{2}, \delta_{i}:=\operatorname{gcd}\left(z_{i}, \bar{n}_{1}\right)$ and let $t_{i}$ be defined by $z_{i} t_{i} \equiv \delta_{i}\left(\bmod \bar{n}_{1}\right)$ with $\operatorname{gcd}\left(t_{i}, \bar{n}_{1}\right)=1,1 \leq i \leq s$.

(i) Assume that there is an index $2 \leq i \leq s$ such that $\delta_{i}>(\log N)^{s}$. Then we have

$$
R_{N}\left(\mathbf{z}_{1}, \mathbf{z}_{2}\right) \geq \sum_{\substack{l=1 \\ h_{i}=l\left(N / \delta_{i}\right)}}^{\delta_{i}-1} \frac{1}{h_{i}} \geq \frac{\delta_{i}}{N}>\frac{(\log N)^{s}}{N} .
$$

So we may assume in the following that $\delta_{i} \leq(\log N)^{s}$ holds for all $1 \leq i \leq s$.

(ii) Assume that $n_{2}>(\log N)^{s}$. Then we have

$$
R_{N}\left(\mathbf{z}_{1}, \mathbf{z}_{2}\right) \geq \sum_{\substack{l=1 \\ h_{1}=l\left(N / n_{2}\right)}}^{n_{2}-1} \frac{1}{h_{1}} \geq \frac{n_{2}}{N}>\frac{(\log N)^{s}}{N} .
$$

So we may assume in the following that $n_{2} \leq(\log N)^{s}$. 
(iii) Assume that there is an index $2 \leq i \leq s$ such that one of the rationals $\frac{\delta_{i} t_{i}}{\bar{n}_{1}}$ has a continued fraction coefficient $a_{k}^{i}>(\log N)^{s}$. W.l.o.g. assume that $i=2$. Then we have

$$
\begin{aligned}
& R_{N}\left(\mathbf{z}_{1}, \mathbf{z}_{2}\right) \geq \sum_{\substack{-N<h_{1}, h_{2}<N \\
h_{1}+h_{2} z_{2} \equiv 0 \\
h_{1} \zeta_{1}+h_{2} \zeta_{2} \equiv 0}}^{*\left(\bmod n_{1}\right)} \underset{1}{r\left(h_{1}\right) r\left(h_{2}\right)} \\
& \geq \sum_{\substack{-n_{1}<h_{1}, h_{2}<n_{1} \\
h_{1}+h_{2} z_{2} \equiv 0}}^{*} \frac{1}{n_{2} n_{2} r\left(h_{1}\right) r\left(h_{2}\right)} \\
& =\sum_{\substack{-n_{1}<h_{1}, h_{2}<n_{1} \\
h_{1} t_{2}+h_{2} \delta_{2} \equiv 0}}^{*} \frac{1}{n_{2} n_{2} r\left(h_{1}\right) r\left(h_{2}\right)} \\
& \geq \sum_{\substack{-n_{1}<h_{1}, h_{2}<n_{1} \\
h_{1} \equiv 0 \quad\left(\bmod \delta_{2}\right) \\
h_{1} t_{2}+h_{2} \delta_{2} \equiv 0 \quad\left(\bmod \bar{n}_{1}\right)}}^{*} \frac{1}{n_{2} n_{2} r\left(h_{1}\right) r\left(h_{2}\right)} \\
& \geq \sum_{\substack{-\bar{n}_{1} / \delta_{2}<h_{1}, h_{2}<\bar{n}_{1} / \delta_{2} \\
h_{1} t_{2}+h_{2} \equiv 0}}^{*} \frac{1}{n_{2} n_{2} \delta_{2} r\left(h_{1}\right) r\left(h_{2}\right)} .
\end{aligned}
$$

For $h_{1} \in \mathbb{Z}$ let

$$
H\left(h_{1}\right):= \begin{cases}-\frac{\bar{n}_{1}}{\delta_{2}}\left\{h_{1} \frac{\delta_{2} t_{2}}{\bar{n}_{1}}\right\}, & \text { if }\left\{h_{1} \frac{\delta_{2} t_{2}}{\bar{n}_{1}}\right\} \leq \frac{1}{2}, \\ \frac{\bar{n}_{1}}{\delta_{2}}\left(1-\left\{h_{1} \frac{\delta_{2} t_{2}}{\bar{n}_{1}}\right\}\right), & \text { if }\left\{h_{1} \frac{\delta_{2} t_{2}}{\bar{n}_{1}}\right\}>\frac{1}{2} .\end{cases}
$$

Then we have $h_{1} t_{2}+H\left(h_{1}\right) \equiv 0\left(\bmod \bar{n}_{1} / \delta_{2}\right)$ and

$$
\left|H\left(h_{1}\right)\right|=\frac{\bar{n}_{1}}{\delta_{2}}\left\|h_{1} \frac{\delta_{2} t_{2}}{\bar{n}_{1}}\right\| .
$$

(Here and in the following $\|$.$\| denotes the distance to the nearest integer$ function, i.e., $\|x\|=\min (\{x\}, 1-\{x\})$.) Now let

$$
\frac{\delta_{2} t_{2}}{\bar{n}_{1}}=\left[0 ; a_{1}, a_{2}, \ldots, a_{m}\right]
$$

and let $q_{-1}, q_{0}, q_{1}, \ldots, q_{m}$ be the denominators of the convergents of $\frac{\delta_{2} t_{2}}{\bar{n}_{1}}$, $q_{-1}=0, q_{0}=1$ and $q_{l}=a_{l} q_{l-1}+q_{l-2}$ for $1 \leq l \leq m$. Assume that $a_{k}>(\log N)^{s}$. Let $h_{1}:=q_{k-1}$, then we have

$$
R_{N}\left(\mathbf{z}_{1}, \mathbf{z}_{2}\right) \geq \frac{1}{n_{2} n_{2} \delta_{2} q_{k-1}\left|H\left(q_{k-1}\right)\right|}
$$

Since

$$
\frac{\delta_{2} t_{2}}{\bar{n}_{1}}-\frac{p_{k-1}}{q_{k-1}}=\frac{\theta_{k}}{a_{k} q_{k-1}^{2}}
$$

with $\left|\theta_{k}\right|<1$, it follows that

$$
q_{k-1} \frac{\delta_{2} t_{2}}{\bar{n}_{1}}=p_{k-1}+\frac{\theta_{k}}{a_{k} q_{k-1}}
$$


and hence we have

$$
\left|H\left(q_{k-1}\right)\right|=\frac{\bar{n}_{1}}{\delta_{2}}\left\|\frac{\theta_{k}}{a_{k} q_{k-1}}\right\| \leq \frac{\bar{n}_{1}}{\delta_{2} a_{k} q_{k-1}} .
$$

From this we get

$$
R_{N}\left(\mathbf{z}_{1}, \mathbf{z}_{2}\right) \geq \frac{\delta_{2} a_{k} q_{k-1}}{n_{2} n_{2} \delta_{2} q_{k-1} \bar{n}_{1}}=\frac{a_{k}}{N}>\frac{(\log N)^{s}}{N} .
$$

So we may assume in the following that all continued fraction coefficients of the rationals $\frac{\delta_{i} t_{i}}{\bar{n}_{1}}, 2 \leq i \leq s$, are less than or equal to $(\log N)^{s}$.

Moreover we assume $N$ so large that

$$
\log N<2 \log \left(\frac{N}{(\log N)^{3 s}}\right)
$$

For the finitely many $N$ that do not satisfy the last inequality, the assertion of the theorem is trivially true with $c_{s}>0$ small enough.

(iv) Define $d_{1}:=n_{2}$ and for $2 \leq k \leq s$ define $d_{k}:=\operatorname{gcd}\left(z_{k} \zeta_{1}-\zeta_{k}, d_{k-1}\right)$. For $2 \leq k \leq s$ and for $v, w \in \mathbb{Z}$ define

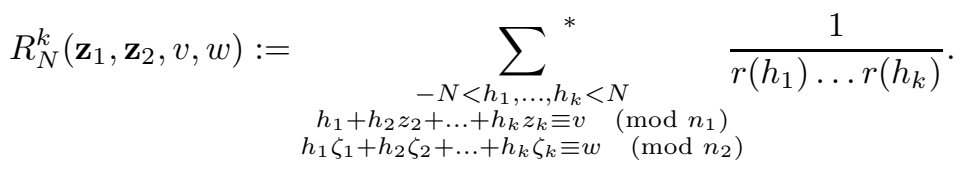

We shall prove that for $v \zeta_{1} \equiv w\left(\bmod d_{k}\right)$ we have

$$
R_{N}^{k}\left(\mathbf{z}_{1}, \mathbf{z}_{2}, v, w\right) \geq c(s, k) d_{k} \frac{(\log N)^{k}}{N}
$$

where $c(s, k)>0$ is a constant depending only on $s$ and $k$ (but not on $N$ ). We do this by induction on $k$.

$k=2:$ Let $v, w \in \mathbb{Z}$ with $v \zeta_{1} \equiv w\left(\bmod d_{2}\right)$ and define

$$
R^{2}:=R_{N}^{2}\left(\mathbf{z}_{1}, \mathbf{z}_{2}, v, w\right)=\sum_{\substack{-N<h_{1}, h_{2}<N \\ h_{1}+h_{2} z_{2} \equiv v \\ h_{1} \zeta_{1}+h_{2} \zeta_{2} \equiv w\left(\bmod n_{1}\right)}}^{*\left(\bmod n_{2}\right)}
$$

For $h_{2} \in \mathbb{Z}$ the system

$$
\begin{aligned}
h_{1}+h_{2} z_{2} & \equiv v\left(\bmod n_{1}\right), \\
h_{1} \zeta_{1}+h_{2} \zeta_{2} & \equiv w\left(\bmod n_{2}\right)
\end{aligned}
$$

has a solution $h_{1}$ iff

$$
h_{2} \zeta_{2} \equiv w \quad\left(\bmod \sigma_{1}\right)
$$

and

$$
h_{2}\left(z_{2} \zeta_{1}-\zeta_{2}\right) \equiv v \zeta_{1}-w \quad\left(\bmod n_{2}\right)
$$

(Here $\sigma_{1}:=\operatorname{gcd}\left(\zeta_{1}, n_{2}\right)$. The second congruence is obtained with Lemma 2.5.) Let $h$ be a solution of congruence (6). Then we have

$$
\zeta_{2} h \equiv w+\zeta_{1}\left(z_{2} h-v\right) \quad\left(\bmod n_{2}\right) .
$$

Now from the definition of $\sigma_{1}$ we obtain $\zeta_{2} h \equiv w\left(\bmod \sigma_{1}\right)$ and so $h$ is also a solution of congruence (5). Hence in the following we only have to consider congruence (6). 
From $v \zeta_{1}-w \equiv 0\left(\bmod d_{2}\right)$ and $d_{2}=\operatorname{gcd}\left(z_{2} \zeta_{1}-\zeta_{2}, n_{2}\right)$ we find that congruence (61) has $d_{2}$ incongruent $\left(\bmod n_{2}\right)$ solutions $x_{1}, \ldots, x_{d_{2}} \in \mathbb{Z}$ with $0 \leq x_{i}<n_{2}$. Now let $i \in\left\{1, \ldots, d_{2}\right\}$ and let $h_{2}=x_{i}+\bar{h}_{2} n_{2}$. Then system (4) becomes

$$
\begin{aligned}
h_{1}+\left(x_{i}+\bar{h}_{2} n_{2}\right) z_{2} & \equiv v\left(\bmod n_{1}\right), \\
h_{1} \zeta_{1}+\left(x_{i}+\bar{h}_{2} n_{2}\right) \zeta_{2} & \equiv w\left(\bmod n_{2}\right) .
\end{aligned}
$$

From congruence (효 we get

$$
h_{1} \zeta_{1} \equiv w-x_{i} \zeta_{2} \quad\left(\bmod n_{2}\right) .
$$

Since $x_{i}$ is a solution of congruence (6) (and hence of congruence (5)), we have $w-x_{i} \zeta_{2} \equiv 0\left(\bmod \sigma_{1}\right)$. Now define $\alpha:=\zeta_{1} / \sigma_{1}, \omega_{i}:=\left(w-x_{i} \zeta_{2}\right) / \sigma_{1}$, $\bar{n}_{2}:=n_{2} / \sigma_{1}$. Then congruence (9) may be rewritten as

$$
h_{1} \alpha \equiv \omega_{i} \quad\left(\bmod \bar{n}_{2}\right) .
$$

Let $\tau_{1} \in \mathbb{Z}$ be defined by $\zeta_{1} \tau_{1} \equiv \sigma_{1}\left(\bmod n_{2}\right)$ with $\operatorname{gcd}\left(\tau_{1}, n_{2}\right)=1$ and define $s_{i}:=\omega_{i} \tau_{1}$. Then we obtain from (10) the congruence $h_{1} \equiv s_{i}$ $\left(\bmod \bar{n}_{2}\right)$ and hence $h_{1}$ is of the form

$$
h_{1}=s_{i}+\bar{h}_{1} \bar{n}_{2}
$$

(w.l.o.g. assume that $0 \leq s_{i}<\bar{n}_{2}$ ). Substituting this in congruence (7), we get

$$
\bar{h}_{1} \bar{n}_{2}+\bar{h}_{2} n_{2} z_{2} \equiv v-s_{i}-x_{i} z_{2} \quad\left(\bmod n_{1}\right) .
$$

Once again we note that $x_{i}$ is a solution of congruence (6), i.e.,

$$
v \zeta_{1}-w-\zeta_{1} z_{2} x_{i}+x_{i} \zeta_{2} \equiv 0 \quad\left(\bmod n_{2}\right) .
$$

By the definition of $\tau_{1}$ we obtain

$$
v \sigma_{1}-\left(w-x_{i} \zeta_{2}\right) \tau_{1}-\sigma_{1} z_{2} x_{i} \equiv 0 \quad\left(\bmod n_{2}\right)
$$

and hence we have $v-s_{i}-z_{2} x_{i} \equiv 0\left(\bmod \bar{n}_{2}\right)$. So we get an integer $a_{i}$ such that $v-s_{i}-z_{2} x_{i}=a_{i} \bar{n}_{2}$. Therefore congruence (11) becomes

$$
\bar{h}_{1}+\bar{h}_{2} \sigma_{1} z_{2} \equiv a_{i} \quad\left(\bmod \sigma_{1} \bar{n}_{1}\right) .
$$

(Recall that $n_{1}=\bar{n}_{1} n_{2}$. ) Now we have

$$
R^{2} \geq \sum_{i=1}^{d_{2}} \sum_{\begin{array}{c}
-N<h_{1}, h_{2}<N \\
h_{2}=x_{i}+\bar{h}_{2} n_{2} \\
h_{1}=\bar{s}_{i}+\bar{h}_{1} \bar{n}_{2} \\
\bar{h}_{1}+\bar{h}_{2} \sigma_{1} z_{2} \equiv a_{i}\left(\bmod \sigma_{1} \bar{n}_{1}\right)
\end{array}}^{*} \frac{1}{r\left(s_{i}+\bar{h}_{1} \bar{n}_{2}\right) r\left(x_{i}+\bar{h}_{2} n_{2}\right)} .
$$

Denote the inner sum in inequality (13) by $\sum_{(1)}(i)$ for $1 \leq i \leq d_{2}$.

Define $\delta:=\sigma_{1} \operatorname{gcd}\left(z_{2}, \bar{n}_{1}\right)=\sigma_{1} \delta_{2}$. From $\bar{h}_{1}+\bar{h}_{2} \sigma_{1} z_{2} \equiv a_{i}\left(\bmod \sigma_{1} \bar{n}_{1}\right)$ it follows that $\bar{h}_{1}=b+l \delta$ for a $b$ with $0 \leq b<\delta$, and $a_{i}-b \equiv 0(\bmod \delta)$; furthermore, $\bar{h}_{2} \sigma_{1} z_{2} \equiv a_{i}-b-l \delta\left(\bmod \sigma_{1} \bar{n}_{1}\right)$. Let $u:=\frac{a_{i}-b}{\delta} t_{2}$. Then $\bar{h}_{2} \equiv u-l t_{2}(\bmod m)$, where $m:=\bar{n}_{1} / \delta_{2}$, and so $\bar{h}_{2}$ is of the form

$$
\bar{h}_{2}=m\left(\frac{u-l t_{2}}{m}+k\right)
$$


where $k \in \mathbb{Z}$. It follows that for every $l \in \mathbb{Z}$ there is a solution $\bar{h}_{1}$ and $\bar{h}_{2}$ of congruence (12) with

$$
\begin{aligned}
\bar{h}_{1} & =b+l \delta \\
\left|\bar{h}_{2}\right| & =m\left\|\frac{u}{m}-l \frac{t_{2}}{m}\right\| .
\end{aligned}
$$

Hence we have

$$
\begin{aligned}
& \sum(i) \geq \sum_{l=0}^{m-1} \frac{1}{\frac{n_{2}}{\sigma_{1}}(b+l \delta+1) n_{2}\left(1+m\left\|\frac{u}{m}-l \frac{t_{2}}{m}\right\|\right)} \\
& \geq \sum_{l=0}^{m-1} \frac{1}{n_{2} \frac{n_{2}}{\sigma_{1}}(\delta(1+l)+1) m\left(\frac{1}{m}+\left\|\frac{u}{m}-l \frac{t_{2}}{m}\right\|\right)} \\
& \geq \sum_{l=0}^{m-1} \frac{1}{n_{2} \frac{n_{2}}{\sigma_{1}} \frac{\bar{n}_{1}}{\delta_{2}} 2 \delta_{2} \sigma_{1}(l+1)\left(\frac{1}{m}+\left\|\frac{u}{m}-l \frac{t_{2}}{m}\right\|\right)} \\
& \geq \frac{1}{4 N} \sum_{l=0}^{m-1} \frac{1}{(l+1) \max \left(\frac{1}{m},\left\|\frac{u}{m}-l \frac{t_{2}}{m}\right\|\right)} \text {. }
\end{aligned}
$$

Since $\operatorname{gcd}\left(t_{2}, \bar{n}_{1}\right)=1$, it follows that $\operatorname{gcd}\left(t_{2}, m\right)=1$. By our assumptions on $n_{2}, N$ and $\delta_{2}$ we get $N=n_{2} n_{2} \delta_{2} m<(\log N)^{3 s} m$ and hence $\log N \leq 2 \log m$. Furthermore, we have that $\frac{t_{2}}{m}=\frac{\delta_{2} t_{2}}{\bar{n}_{1}}$ has continued fraction coefficients $a_{i}<(\log N)^{s} \leq 2^{s}(\log m)^{s}$. But under these assumptions G. Larcher proved in [4, p. 48, inequality $(*)$ ] that

$$
\sum_{l=0}^{m-1} \frac{1}{(l+1) \max \left(\frac{1}{m},\left\|a-l \frac{t_{2}}{m}\right\|\right)} \geq c(s)(\log m)^{2}
$$

holds for every $a \in[0,1)$. (Here $c(s)>0$ is a constant depending only on s.) So we get

$$
\sum(i) \geq \frac{1}{4 N} c(s)(\log m)^{2} \geq \frac{c(s)}{8} \frac{(\log N)^{2}}{N} .
$$

Inserting this in inequality (13), we get

$$
R^{2} \geq c(s, 2) d_{2} \frac{(\log N)^{2}}{N},
$$

such that the case $k=2$ is proved.

$k-1 \longrightarrow k$ : For short we write $R^{k}(v, w)$ instead of $R_{N}^{k}\left(\mathbf{z}_{1}, \mathbf{z}_{2}, v, w\right)$. Let $v \zeta_{1} \equiv w\left(\bmod d_{k}\right)$. Then we have

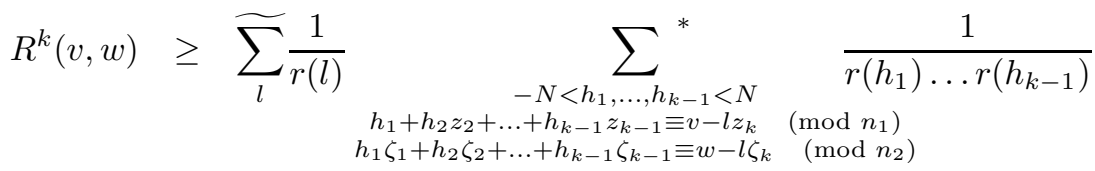

$$
\begin{aligned}
& =\widetilde{\sum_{l}} \frac{1}{r(l)} R^{k-1}\left(v-l z_{k}, w-l \zeta_{k}\right),
\end{aligned}
$$


where $\widetilde{\sum_{l}}$ denotes summation over all integers $-N<l<N$ such that

$$
\left(v-l z_{k}\right) \zeta_{1} \equiv w-l \zeta_{k} \quad\left(\bmod d_{k-1}\right) .
$$

Now we get from the induction hypothesis that

$$
R^{k}(v, w) \geq c(s, k-1) d_{k-1} \frac{(\log N)^{k-1}}{N} \sum_{l} \frac{1}{r(l)} .
$$

Since by our assumption $d_{k}=\operatorname{gcd}\left(z_{k} \zeta_{1}-\zeta_{k}, d_{k-1}\right)$ is a divisor of $v \zeta_{1}-w$, we find $d_{k}$ incongruent solutions $x_{1}, \ldots, x_{d_{k}}$ of congruence (14), $0 \leq x_{i}<d_{k-1}$. Now we have

$$
\begin{aligned}
\widetilde{\sum_{l}} \frac{1}{r(l)} & \geq \sum_{i=1}^{d_{k}} \sum_{\substack{l=0 \\
l=x_{i}+\bar{l} d_{k-1}}}^{N-1} \frac{1}{r\left(x_{i}+\bar{l} d_{k-1}\right)} \geq \sum_{i=1}^{d_{k}} \sum_{\bar{l}=0}^{N / d_{k-1}-1} \frac{1}{(\bar{l}+1) d_{k-1}} \\
& \geq \frac{d_{k}}{d_{k-1}} \log \frac{N}{d_{k-1}} \geq \frac{1}{2} \frac{d_{k}}{d_{k-1}} \log N,
\end{aligned}
$$

since $d_{k-1} \leq d_{1}=n_{2}$ and hence

$$
\log \frac{N}{d_{k-1}} \geq \log \frac{N}{n_{2}}=\log n_{1} \geq \frac{1}{2} \log N .
$$

Inserting this result in (15) will finish our induction proof of inequality (3). The result follows.

Problem 2.6. (1) It remains an open question whether Theorem 2.1 holds without the existence of an index $1 \leq i_{0} \leq s$ such that $\operatorname{gcd}\left(z_{i_{0}}, n_{1}\right)=1$.

(2) Is the lower bound from Theorem 2.1 also true for rank $r$ lattice rules, $2<r \leq s ?$

\section{REFERENCES}

[1] Hlawka, E.: Zur angenäherten Berechnung mehrfacher Integrale. Monatsh. Math. 66: 140151, 1962. MR 26:888

[2] Korobov, N.M.: Numbertheoretical Methods in Approximate Analysis. Moscow: Fizmatgiz. 1963. (In Russian.) MR 28:716

[3] Kuipers, L., Niederreiter, H.: Uniform Distribution of Sequences. John Wiley, New York, 1974. MR 54:7415

[4] Larcher, G.: A Best Lower Bound for Good Lattice Points. Monatsh. Math. 104: 45-51, 1987. MR 89f: 11103

[5] Niederreiter, H.: Existence of Good Lattice Points in the Sense of Hlawka. Monatsh. Math. 86: 203-219, 1978. MR 80e:10039

[6] Niederreiter, H.: The Existence of Efficient Lattice Rules for Multidimensional Numerical Integration. Math. Comp. 58: 305-314 and S7-S16, 1992. MR 92e:65023

[7] Niederreiter, H.: Random Number Generation and Quasi-Monte Carlo Methods. No. 63 in CBMS-NSF Series in Applied Mathematics. SIAM, Philadelphia, 1992. MR 93h:65008

[8] Niederreiter, H., Sloan, I.H.: Lattice rules for multiple integration and discrepancy. Math. Comp. 54: 303-312, 1990. MR 90f:65036

[9] Sloan, I.H., Joe, S.: Lattice Methods for Multiple Integration. Oxford Univ. Press, New York and Oxford, 1994. MR 98a:65026

[10] Sloan, I.H., Lyness, J.N.: Lattice rules: Projection regularity and unique representations. Math. Comp. 54: 649-660, 1990. MR 91a:65062

Institut für Analysis, Universität Linz, Altenbergerstrasse 69, A-4040 Linz, Austria E-mail address: friedrich.pillichshammer@jku.at 